\title{
Investigación sobre tablas y gráficos estadísticos en libros de texto de educación primaria de Iberoamérica: revisión de literatura
}

\author{
Research on statistical tables and graphs in primary education \\ textbooks in Latin America: literature review. \\ Pesquisa sobre tabelas e gráficos estatísticos em livros didáticos \\ do ensino fundamental na Ibero-América: revisão de literatura.
}

\author{
Stiven R. Vidal-Henry \\ Institución Educativa No. 13, Sede Erika Beatriz \\ Maicao, Colombia \\ stvnrvh1993@hotmail.com \\ (D) ORCID: https://orcid.org/0000-0002-2566-4511 \\ Elizabeth H. Arredondo \\ Universidad de Los Lagos \\ Osorno, Chile \\ elizabeth.hernandez@ulagos.cl \\ (D) ORCID: https://orcid.org/0000-0002-5285-1603 \\ Jaime I. García-García \\ Universidad de Los Lagos \\ Osorno, Chile \\ jaime.garcia@ulagos.cl \\ (D) ORCID: https://orcid.org/0000-0002-8799-5981
}

Recibido - Received - Recibido: 10 / 07/ 2021 Corregido - Revised - Revisado: 31 / 10 / 2021 Aceptado - Accepted - Aprobado: 09 / 11 / 2021

DOI: https://doi.org/10.22458/ie.v23i35.3636

URL: https://revistas.uned.ac.cr/index.php/innovaciones/article/view/3636

\begin{abstract}
Resumen: En este artículo se reporta una revisión de literatura respecto a las investigaciones sobre tablas y gráficos estadísticos en libros de texto de educación primaria de Iberoamérica, con el objetivo de entregar al lector un panorama de lo desarrollado y algunas implicaciones a futuro. Bajo una metodología cualitativa, se realizó una revisión sistemática de literatura de los últimos 15 años, en cinco etapas: 1) planeación, 2) búsqueda, 3) selección, 4) evaluación de calidad, y 5) extracción y síntesis. La búsqueda se realizó en la base de datos de libre acceso Education Resources Information Center (ERIC) y en los buscadores Google Scholar y Semantic Scholar. De los resultados de la búsqueda, se seleccionaron 39 investigaciones: 29 publicadas en revistas y 10 en memorias de congresos. Entre los hallazgos, el diagrama geográfico evidencia una amplia exploración y concentración de la línea investigativa solo en algunos países, con un enfoque en la exploración de la calidad de las actividades y/o en identificar el tipo de representación estadística usada y sus complejidades para resolver las tareas. Además, se identifica una gran variedad de unidades de análisis empleadas para el desarrollo de estas investigaciones, situándolas en analizar el nivel de lectura, el nivel de complejidad semiótica, la tarea solicitada y el tipo de contexto de las actividades en las que aparecen las tablas y gráficos estadísticos.
\end{abstract}

Palabras clave: tabla estadística, gráfico estadístico, estadística, libro de texto, educación básica.

Summary: This article reports a literature review regarding research on statistical tables and graphs in primary school textbooks in Latin America, with the aim of providing the reader with an overview of what has been developed and some implications for the future. Under a qualitative methodology, a systematic review of the literature of the last 15 years was carried out, in five stages: 1) planning, 2) search, 3) selection, 4) quality evaluation, and 5) extraction and synthesis. The search was carried out in the open access database Education Resources Information Center (ERIC) and in the search engines Google Scholar and Semantic Scholar. From the search results, 39 investigations were selected: 29 published in journals and 10 in conference memoires. Among the findings, the geographic diagram shows a broad exploration and concentration of the research line only 
in some countries, with a focus on exploring the quality of activities and/or identifying the type of statistical representation used and its complexities to resolve tasks. In addition, a great variety of analysis units used for the development of these investigations is identified, placing them in analyzing the reading level, the level of semiotic complexity, the requested task and the type of context of the activities in which the tables and statistical graphics appear.

Key Words: statistical table, statistical graph, statistics, textbook, basic education.

Resumo: Este artigo apresenta uma revisão bibliográfica de pesquisas sobre tabelas e gráficos estatísticos em livros escolares do ensino fundamental na Ibero-América, com o objetivo de proporcionar ao leitor uma visão geral do que foi desenvolvido e algumas implicações para o futuro. Sob uma metodologia qualitativa, foi realizada uma revisão sistemática da literatura dos últimos 15 anos em cinco etapas: 1) planejamento, 2) busca, 3) seleção, 4) avaliação da qualidade, e 5) extração e síntese. A pesquisa foi realizada no banco de dados de acesso aberto Education Resources Information Center (ERIC) e nos mecanismos de busca Google Scholar and Semantic Scholar. Dos resultados da pesquisa, foram selecionados 39 trabalhos de pesquisa: 29 publicados em revistas e 10 em anais de conferências. Entre as conclusões, o diagrama geográfico apresenta uma ampla exploração e concentração da linha de pesquisa em apenas alguns países, com foco na exploração da qualidade das atividades e/ou identificação do tipo de representação estatística utilizada e suas complexidades na resolução das tarefas. Além disso, é identificada uma grande variedade de unidades de análise empregadas para o desenvolvimento dessas investigações, colocando-as na análise do nível de leitura, do nível de complexidade semiótica, da tarefa solicitada e do tipo de contexto das atividades em que as tabelas e gráficos estatísticos aparecem.

Palavras-chave: tabela estatística, gráfico estatístico, estatística, livro-texto, educação fundamental.

\section{INTRODUCCIÓN}

En la actualidad existe un creciente interés por explorar la enseñanza y el aprendizaje de la estadística en educación primaria, cuya inclusión en el currículo escolar ha sido paulatina desde hace veinticinco años, como lo evidencian las directrices curriculares de diversos países (Vásquez y Alsina, 2014), con el propósito de fomentar la "cultura estadística". Este constructo cada día se incrusta más como agenda en los rediseños curriculares escolares; se refiere a la capacidad de interpretar y evaluar críticamente información que se puede encontrar en distintas representaciones estadísticas, y a la capacidad para discutir y comunicar tal información (Gal, 2002). En este sentido, las tablas y los gráficos estadísticos son considerados como parte de este constructo (Arteaga, Batanero, Cañadas y Contreras, 2011; Batanero, 2004; Del Pino y Estrella, 2012; Gal, 2002); cuya lectura e interpretación son habilidades que debe desarrollar el estudiantado durante su educación básica para ser capaces de comprender la información a la que tienen acceso a través de diversos medios de comunicación (televisión, internet, revistas, entre otros) y, con esto, ser parte del progreso de una sociedad estadísticamente culta (Contreras y MolinaPortillo, 2019).

La exploración del libro de texto como informante del estado de la evolución del proceso de enseñanzaaprendizaje de la matemática ha ido en aumento, justificada en su papel fundamental en dicho proceso (Ferreira y Mayorga, 2010; Rodríguez, 2007), en vista de que en este se propone un trabajo sistemático y continuo de los contenidos propuestos en el currículo escolar (Son y Diletti, 2017). Además, el libro de texto es considerado como un instrumento necesario en el desarrollo de la práctica docente (Claros, Sánchez y Coriat, 2016) y como una herramienta para el quehacer didáctico de dicha práctica (Soaje de Elias, 2018), motivos por los cuales algunos autores proponen su análisis como parte de un proceso que permite enriquecer los contenidos que este presenta, puesto que en este recurso educativo se encuentran fortalezas o debilidades (Fernández y Caballero, 2017).

La revisión de la literatura se encuentra ligada estrechamente con los objetivos y propósitos de la investigación, puesto que permite refinar el problema a investigar detallada y críticamente. También, desde la recopilación y síntesis de la información, da a conocer de manera amplia el conocimiento dado en un periodo de tiempo sobre algún tópico en particular (Guirao, 2015). Ahora bien, entre las revisiones de la literatura se encuentra la revisión sistemática, la cual se realiza basándose en una pregunta 
de investigación y sigue un protocolo para identificar, evidenciar y resumir los estudios primarios relacionados con el objeto de estudio (Moreno-Gómez, 2021).

Entre las líneas de investigación en educación estadística se encuentra el análisis del libro de texto tomando como objeto de estudio la tabla y/o gráfico estadístico, y en este sentido, como informante para explorar el fomento de las habilidades necesarias para el desarrollo de la cultura estadística. Dentro de esta línea de investigación, que tiene más de 15 años tomando fuerza en países iberoamericanos, el presente artículo reporta una revisión sistemática de literatura de las investigaciones sobre tablas y gráficos estadísticos en libros de texto de educación primaria de lberoamérica, con el objetivo de entregar al lector un panorama de lo desarrollado y algunas implicaciones a futuro. En concreto, como objetivos específicos, se analiza: 1) el objetivo de las investigaciones del estudio; 2) las características que se han estudiado; y 3) los resultados relevantes encontrados.

\section{Literatura sobre el tema}

Las investigaciones sobre la enseñanza y aprendizaje de conceptos estadísticos han ido aumentando y tomando relevancia por su inclusión desde los primeros grados de escolaridad (García-García, DíazLevicoy, Vidal-Henry y Arredondo, 2019); una arista de estas se sitúa en el análisis de libros de texto tomando como centro de atención las tablas y los gráficos estadísticos.

Una tabla estadística es un arreglo rectangular con una estructura que contiene un conjunto de filas y columnas, de modo que permite presentar los datos correspondientes a una o más variables en forma clasificada y resumida, permitiendo visualizar el comportamiento de los datos y facilitar la comprensión de la información que se puede extraer (Estrella, 2014). Mientras que, de acuerdo con Curcio (1989), un gráfico estadístico proporciona un medio para clasificar, organizar, resumir y comunicar información cuantitativa, permitiendo comparar datos y mostrar relaciones que comúnmente no pueden reconocerse.

Dentro de la investigación en educación estadística, se han desarrollado diversas jerarquías para analizar la lectura de gráficos, siendo la taxonomía de Curcio (Curcio, 1989; Friel et al, 2001; Shaughnessy, 2007) la más utilizada por diversos autores (García-García, Encarnación y Arredondo, 2020): a) nivel 1, leer los datos, consiste la lectura literal de datos representados en una tabla o un gráfico; b) nivel 2, leer dentro de los datos, implica la interpretación e integración de datos que no se encuentran explícitamente en la tabla o gráfico; c) nivel 3, leer más allá de los datos, conlleva a la realización de inferencias o predicciones con los datos mostrados en la tabla o gráfico; y d) nivel 4, leer detrás de los datos, consiste en una valoración crítica de los datos y del tipo de representación utilizada, así como la integración de la información con el contexto para realizar conclusiones. Ahora bien, con respecto a la construcción de un gráfico estadístico, Arteaga y colaboradores (Arteaga, 2011; Batanero, Arteaga y Ruiz, 2010) definen niveles de complejidad semiótica: a) nivel 1, representación de datos aislados, sin realizar una representación conjunta; b) nivel 2, representación de una serie de valores de una variable, sin llegar a resumir su distribución; c) nivel 3, representación de una distribución de datos; y d) nivel 4, representación de varias distribuciones sobre una misma representación. Ambos niveles jerárquicos son aplicables a las tablas.

Con respecto a estas representaciones estadísticas, en Arteaga, Díaz-Levicoy y Batanero (2018) se presenta una revisión de literatura acerca de las investigaciones sobre la lectura y construcción de gráficos por parte del estudiantado de educación primaria. Este estudio se apoya en la elección por conveniencia de algunos artículos, los cuales son analizados bajo algunas taxonomías, ampliamente estudiadas en el campo, que permiten identificar el nivel de lectura. Sin embargo, a pesar de la interesante propuesta, la elección de los artículos no aporta una visión holística acerca del estado del arte de la investigación desarrollada sobre la construcción y lectura de gráficos estadísticos por el estudiantado de países de Iberoamérica, o bien, donde se use como elemento de análisis los libros de texto de educación 
primaria. En ese sentido, se identifica un área de oportunidad en el desarrollo de un estudio de estas características, que reporte la investigación desarrollada sobre gráficos y tablas estadísticas en libros de texto iberoamericanos; esto puede llevar a identificar la distribución geográfica de las investigaciones, los objetivos de estas y los resultados más relevantes.

\section{MÉTODO}

Este artículo sintetiza el estado del arte que guardan las investigaciones del estudio publicadas en revistas y memorias de congreso, 2006 y hasta junio 2021, mismas que pasaron por una revisión de pares.

\section{Recolección de datos y selección de estudios a analizar}

Para la revisión sistemática de literatura se consideró la base de datos Education Resources Information Center (ERIC) y los buscadores Google Scholar y Semantic Scholar, con criterios de búsqueda y selección que se abordarán más adelante. De acuerdo con Carrizo y Ortiz (2016): “Una revisión sistemática de literatura es una metodología de investigación que se desarrolla para obtener y evaluar la evidencia disponible que sea pertinente sobre un tema específico" (p. 188). Bajo esta perspectiva, en este artículo se considera una revisión sistemática de literatura como una síntesis de aspectos cualitativos de artículos existentes respecto a las investigaciones del estudio, que permite sintetizar los resultados de los estudios primarios para contribuir a la línea de investigación en educación estadística.

El método empleado para la selección de literatura se desarrolló en cinco etapas (ver Figura 1), considerando las propuestas por Revelo, Collazos y Jiménez (2018).

FIGURA 1

Esquema del método de revisión sistemática de literatura empleado.

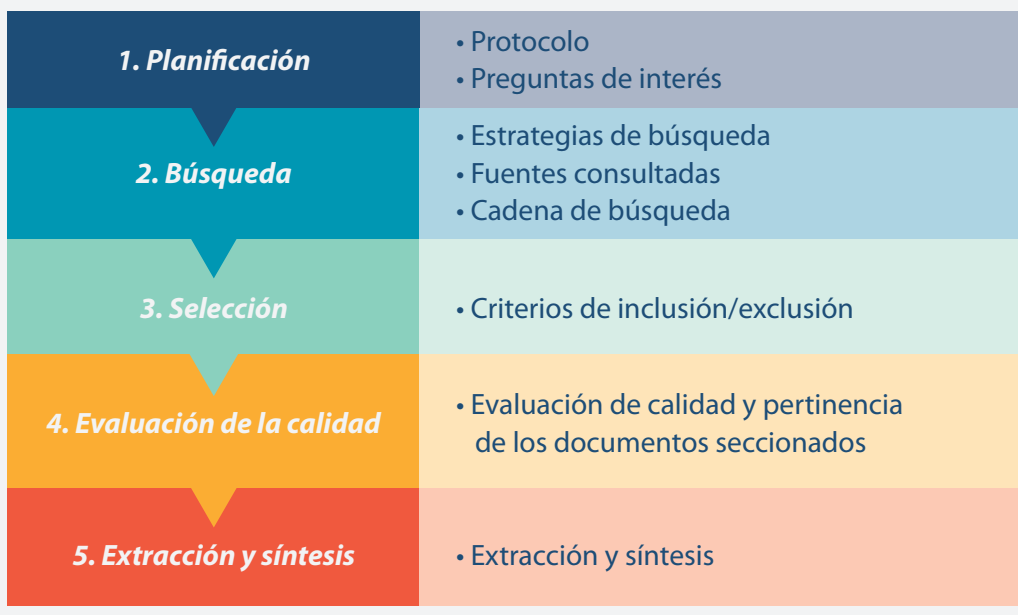

Fuente: Elaboración propia (adaptado de Revelo, Collazos y Jiménez, 2018).

Etapa 1: Planeación. Se enfoca en desarrollar un protocolo para la revisión sistemática. Se detalla el alcance de la revisión y las acciones a seguir para lograr los objetivos. En este estudio se establecieron las preguntas de interés, las estrategias de búsqueda, los criterios de inclusión/exclusión y de evaluación de 
calidad, y los procedimientos para la extracción y síntesis de datos. Las preguntas de interés se presentan a continuación, y los otros elementos del protocolo se describen en las etapas siguientes.

P1: ¿Cuál es el objetivo de las investigaciones sobre tablas y gráficos estadísticos en libros de texto de educación primaria de lberoamérica?

P2: ¿Qué características se han estudiado en las investigaciones sobre tablas y gráficos estadísticos en libros de texto de educación primaria de lberoamérica?

P3: ¿Cuáles son los resultados relevantes encontrados en las investigaciones rastreadas?

Etapa 2: Búsqueda. Se centra en un proceso de búsqueda exhaustivo e imparcial, cuyo factor principal es identificar las palabras claves vinculadas al tema de interés del estudio, y con ello, realizar cadenas de búsqueda en distintas fuentes. En este caso, los recursos utilizados fueron: la base de datos Education Resources Information Center (ERIC) y los motores de búsqueda Google Scholar y Semantic Scholar; mientras que las palabras clave fueron: tablas estadísticas, gráficos estadísticos, libros de texto y educación primaria, y las cadenas de búsqueda utilizadas fueron: tablas estadísticas en libros de texto de educación primaria, gráficos estadísticos en libros de texto de educación primaria, tablas estadísticas en actividades de libros de texto de educación primaria, gráficos estadísticos en actividades de libros de texto de educación primaria, entre otras. Cabe señalar que la búsqueda se realizó en español y en inglés, apoyándonos en operadores boolenaos (and, or y not).

Etapa 3: Selección. Consiste en la selección preliminar de los documentos a partir de la lectura y revisión del título y resumen, con el fin de escoger las investigaciones relacionadas con el estudio. Posteriormente, se realiza una selección detallada con base en una lectura preliminar del texto completo de las investigaciones seleccionadas. En esta etapa de la revisión de literatura se obtuvieron 65 investigaciones.

Etapa 4: Evaluación de calidad. Consiste en evaluar los documentos seleccionados de acuerdo criterios (según el tema de interés) para asegurar la calidad y pertinencia de la revisión sistemática. En este caso, con relación a la calidad, se evaluó la relevancia y claridad de la investigación para dar respuesta a las preguntas de interés, considerando el objetivo y la descripción del contexto del estudio. Al mismo tiempo, en cuanto a la pertinencia, se revisaron las unidades de análisis y los libros de texto considerados por país para evitar la duplicación de investigaciones, elaboradas por el(los) mismo(s) autor(es) al presentar los resultados inicialmente en memorias de congresos y después en revistas. A partir de esta evaluación, se llegó a 39 investigaciones que sirvieron como estudios primarios para la revisión sistemática de la literatura, cuyas referencias se presentan en la sección de anexo.

Etapa 5, extracción y síntesis. Consiste en la extracción de datos relevantes de los estudios primarios con el propósito de responder las preguntas de interés planteadas, y en la síntesis de los resultados obtenidos. En este caso, de cada documento seleccionado se extrajeron los siguientes datos: lugar de publicación, año de publicación, tipo de representación estadística analizada, país de los libros de texto analizados, objetivo de la investigación, unidades y categorías de análisis, y los resultados relevantes. Para la síntesis de los datos obtenidos, se elaboraron tablas de registro. 


\section{DISCUSIÓN DE RESULTADOS}

A continuación, en la Tabla 1 se muestra un resumen cuantitativo de las investigaciones seleccionadas por lugar de publicación. Se observa que la mayoría de las investigaciones (24 de 39) toman como objeto de estudio los gráficos estadísticos, así como una mayor proporción de estas (29 de 39) fueron publicadas en revistas, lo que evidencia la consolidación de esta línea de investigación en educación estadística.

TABLA 1

Clasificación de las investigaciones seleccionadas por lugar de publicación.

\begin{tabular}{|l|c|c|c|c|}
\hline \multicolumn{1}{|c|}{ Lugar de publicación } & Gráficos (G) & Tablas (T) & $\begin{array}{c}\text { Gráficos y } \\
\text { tablas (GT) }\end{array}$ & Total \\
\hline Revistas & 19 & 9 & 1 & 29 \\
\hline Memorias de congresos & 5 & 2 & 3 & 10 \\
\hline Total & $\mathbf{2 4}$ & $\mathbf{1 1}$ & $\mathbf{4}$ & $\mathbf{3 9}$ \\
\hline
\end{tabular}

Fuente: Elaboración propia.

En la Tabla 2 se enlistan las investigaciones seleccionadas por año de publicación. Se observa que las investigaciones del estudio tienen cabida desde hace 15 años, masificándose en el último lustro.

TABLA 2

Investigaciones seleccionadas por año de publicación.

\begin{tabular}{|l|l|}
\hline Año & \multicolumn{1}{|c|}{ Estudio primario (País iberoamericano) } \\
\hline 2006 & G01 (Brasil) \\
\hline 2007 & GT01 (Brasil) \\
\hline 2008 & GT02 (Brasil) \\
\hline 2011 & GT03 (Brasil) \\
\hline 2013 & G03 (Brasil), G02 (España) \\
\hline 2014 & G04 (Colombia), G05 (Chile) \\
\hline 2015 & G07 (España), G06 (Chile) \\
\hline 2016 & T02 (Brasil), G11 (España), G12, G13 y T01 (Chile), G08 (Venezuela), G09 (Venezuela y Guatemala), \\
\hline 2017 & G10 (España y Chile) \\
\hline 2018 & G16 (España), G15 y T03 (Chile), G14 (Argentina) \\
\hline 2019 & T06 (Chile), G17 y G18 (Perú) \\
\hline 2020 & G20, G21, T09 y T10 (Chile), T08 (Venezuela), G22 (Costa Rica), G23 (Brasil y Chile), G24 (México) \\
\hline 2021 & T11 (Chile) \\
\hline
\end{tabular}

Fuente: Elaboración propia con base en la revisión sistemática.

En el Figura 1 se muestra el perfil geográfico del desarrollo y concentración de las investigaciones. Se reconoce que los libros de texto de educación primaria de Chile (16 estudios primarios) y Brasil (8 estudios primarios) han sido los más analizados. Además, se identifican los países donde las investigaciones del estudio ha sido poca o nula; esto abre líneas de acción para aquellos que se interesen en seguir explorando. Por otro lado, algo interesante a explorar sería: ¿cuál ha sido el impacto de estas investigaciones en las políticas educativas? ¿los resultados de estas investigaciones se han utilizado para diseñar o rediseñar los libros de texto?, ¿por qué el poco o nulo interés por explorar en algunos países?, ¿será que la importancia de la cultura estadística no ha impactado los currículos de estos países 
FIGURA 2

Presencia de las investigaciones del estudio.

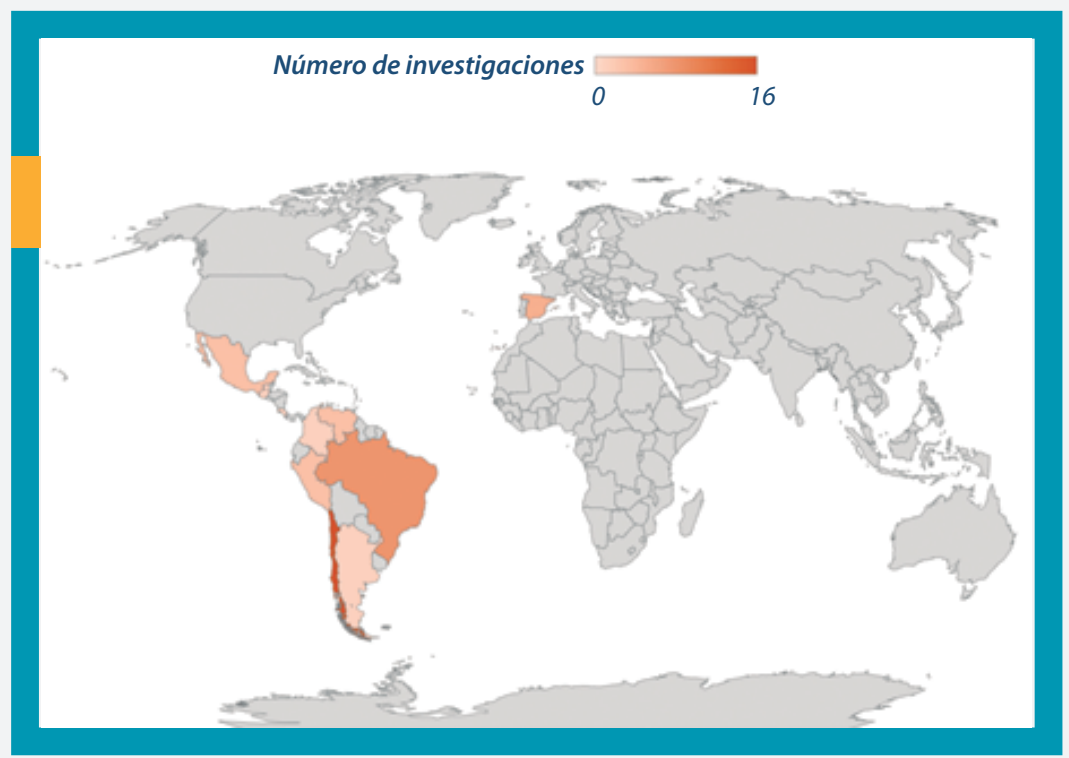

Fuente: Elaboración propia con base en la revisión sistemática.

Con el propósito de responder la pregunta P1: ¿Cuál es el objetivo de las investigaciones sobre tablas y gráficos estadísticos en libros de texto de educación primaria de Iberoamérica?, en la Tabla 3 se presentan los objetivos de las investigaciones seleccionadas. Como se observa, en la mayoría de ellas se declara el verbo analizar (26 de 39).

TABLA 3

Objetivo de las investigaciones seleccionadas.

\begin{tabular}{|c|l|}
\hline Código & \multicolumn{1}{|c|}{ Objetivo de la investigación } \\
\hline G01 & $\begin{array}{l}\text { Investigar el tratamiento de la información expresada en gráficos estadísticos en los libros de } \\
\text { texto brasileños presentados en tres colecciones. }\end{array}$ \\
\hline G02 & $\begin{array}{l}\text { Analizar la presencia del contenido de gráficos estadísticos en las directrices curriculares } \\
\text { oficiales españolas y la forma en que se trabaja dicho contenido en una serie de libros de } \\
\text { matemáticas de educación primaria. Comparativo curricular. }\end{array}$ \\
\hline G03 & $\begin{array}{l}\text { Analizar el tipo, la calidad de los gráficos y el nivel de comprensión requerido para su lectura e } \\
\text { interpretación. }\end{array}$ \\
\hline $\mathbf{G 0 4}$ & $\begin{array}{l}\text { Estudiar los gráficos estadísticos, presentes en algunos libros de matemáticas escolares } \\
\text { publicados en el periodo de 1960 al 2010, en Bogotá. Comparativo curricular. }\end{array}$ \\
\hline $\mathbf{G 0 5}$ & $\begin{array}{l}\text { Analizar las actividades en las que intervienen gráficos estadísticos, en dos libros de texto para } \\
\text { séptimo año de educación básica chilena. }\end{array}$ \\
\hline $\mathbf{G 0 6}$ & $\begin{array}{l}\text { Analizar las variables que determinan los gráficos estadísticos en los textos de educación } \\
\text { primaria en Chile. }\end{array}$ \\
\hline $\mathbf{G 0 7}$ & $\begin{array}{l}\text { Analizar la presentación de los gráficos estadísticos en tres series de libros de textos para la } \\
\text { educación primaria española. }\end{array}$ \\
\hline G08 & $\begin{array}{l}\text { Analizar de las actividades sobre gráficos estadísticos propuestas para el estudiantado que se } \\
\text { encuentran en los libros de matemática la educación primaria y secundaria. }\end{array}$ \\
\hline $\mathbf{G 0 9}$ & $\begin{array}{l}\text { Analizar las actividades propuestas para el tema de gráficos estadísticos para la educación } \\
\text { primaria en Guatemala y Venezuela. }\end{array}$ \\
\hline
\end{tabular}




\begin{tabular}{|c|c|}
\hline Código & Objetivo de la investigación \\
\hline G10 & $\begin{array}{l}\text { Comparar las características de los gráficos estadísticos en libros de texto para la educación } \\
\text { primaria en España y Chile. }\end{array}$ \\
\hline G11 & $\begin{array}{l}\text { Analizar la presencia de los gráficos estadísticos en libros de texto digitales de educación } \\
\text { primaria en España. }\end{array}$ \\
\hline G12 & $\begin{array}{l}\text { Indagar sobre los potenciales conflictos semióticos que aparecen en los libros de texto sobre los } \\
\text { gráficos estadísticos. }\end{array}$ \\
\hline G13 & $\begin{array}{l}\text { Analizar actividades en las que se emplean gráficos estadísticos en libros de texto chilenos de } \\
1^{\circ} \text { a } 6^{\circ} \text { año de educación primaria para la asignatura de ciencias naturales. }\end{array}$ \\
\hline G14 & $\begin{array}{l}\text { Estudiar la presencia de los gráficos estadísticos en cuatro series de libros de texto (12 textos) } \\
\text { para el segundo ciclo de educación primaria en Argentina. }\end{array}$ \\
\hline G15 & $\begin{array}{l}\text { Caracterizar los conflictos semióticos potenciales que aparecen en los gráficos estadísticos } \\
\text { que se encuentran en una muestra de libros de texto para el área de ciencias naturales en } \\
\text { educación primaria chilena. }\end{array}$ \\
\hline G16 & $\begin{array}{l}\text { Mostrar un tipo de análisis (ontosemiótico) sobre los objetos y significados matemáticos que se } \\
\text { movilizan en el enunciado de dos tareas sobre gráficos estadísticos. }\end{array}$ \\
\hline G17 & $\begin{array}{l}\text { Aproximarse al significado institucional presentado sobre los gráficos estadísticos en libros de } \\
\text { matemáticas de educación primaria en Perú. }\end{array}$ \\
\hline G18 & $\begin{array}{l}\text { Estudiar cómo se implementan los gráficos estadísticos en tres series de los libros de educación } \\
\text { primaria en Perú. }\end{array}$ \\
\hline G19 & $\begin{array}{l}\text { Analizar las actividades en las que intervienen gráficos de barras en libros de texto de } \\
\text { matemática de educación primaria en Perú. }\end{array}$ \\
\hline G20 & $\begin{array}{l}\text { Analizar las actividades sobre gráficos estadísticos en los libros de texto diseñados para las } \\
\text { escuelas rurales multigrado. }\end{array}$ \\
\hline G21 & $\begin{array}{l}\text { Analizar actividades evaluativas del libro de texto del profesorado para la educación primaria } \\
\text { rural multigrado que involucran gráficos estadísticos. }\end{array}$ \\
\hline G22 & $\begin{array}{l}\text { Analizar la forma en que se consideran los gráficos en algunas series de libros de texto de } \\
\text { educación primaria en Costa Rica, en los diferentes niveles educativos. }\end{array}$ \\
\hline G23 & $\begin{array}{l}\text { Analizar actividades sobre gráficos estadísticos en libros de texto de matemáticas para el } \\
\text { segundo año de la escuela primaria en Brasil y Chile. }\end{array}$ \\
\hline G24 & $\begin{array}{l}\text { Analizar el nivel de lectura, el nivel de complejidad y el conflicto semiótico de los gráficos } \\
\text { estadísticos presentes en los libros de texto de matemática de educación primaria en México. }\end{array}$ \\
\hline T01 & $\begin{array}{l}\text { Analizar las actividades en las que intervienen tablas estadísticas en los libros de texto de } 1^{\circ} \text { y } \\
2^{\circ} \text { año de educación primaria en Chile. }\end{array}$ \\
\hline T02 & $\begin{array}{l}\text { Observar cómo se presenta y utiliza la tabla estadística en los libros de texto de los primeros } \\
\text { años en Brasil. }\end{array}$ \\
\hline T03 & $\begin{array}{l}\text { Analizar las actividades en las que intervienen tablas estadísticas en los libros de texto de } \\
\text { tercer año de educación primaria. }\end{array}$ \\
\hline T04 & $\begin{array}{l}\text { Analizar las actividades en las que intervienen tablas estadísticas en los libros de texto de } \\
\text { tercer año de educación primaria en Chile. }\end{array}$ \\
\hline T05 & $\begin{array}{l}\text { Identificar el tipo de tabla y el tipo de acción que se plantea al estudiantado en las actividades } \\
\text { propuestas sobre tablas estadísticas. }\end{array}$ \\
\hline T06 & $\begin{array}{l}\text { Analizar actividades que involucren tablas propuestas en colecciones de libros de texto } \\
\text { brasileños del } 1^{\circ} \text { al } 5^{\circ} \text { año de primaria. }\end{array}$ \\
\hline T07 & $\begin{array}{l}\text { Analizar las actividades sobre tablas estadísticas presentes en los libros de texto de educación } \\
\text { primaria en México. }\end{array}$ \\
\hline T08 & $\begin{array}{l}\text { Analizar las actividades de tablas estadísticas de una colección textos escolares de matemáticas } \\
\text { de Venezuela. }\end{array}$ \\
\hline
\end{tabular}




\begin{tabular}{|c|l|}
\hline Código & \multicolumn{1}{|c|}{ Objetivo de la investigación } \\
\hline T09 & $\begin{array}{l}\text { Analizar la actividad algebraica requerida en el manejo de las tablas estadísticas que se tienen } \\
\text { en cuenta en los cursos } 5^{\circ} \text { a } 8^{\circ} \text { de la educación básica y en la educación media en Chile (10 a } 13 \\
\text { años). }\end{array}$ \\
\hline T10 & $\begin{array}{l}\text { Adaptar el constructo complejidad semiótica y analizar la distribución de estos tipos de tablas } \\
\text { estadísticas en una muestra de textos chilenos de educación básica. }\end{array}$ \\
\hline T11 & $\begin{array}{l}\text { Caracterizar de las tareas propuestas sobre tablas estadísticass en libros de texto chilenos de } \\
\text { educación básica. }\end{array}$ \\
\hline GT01 & $\begin{array}{l}\text { Analizar las actividades relacionadas con las representaciones gráficos y tablas y los tipos de } \\
\text { orientaciones didácticas se presentan en el manual del profesor. }\end{array}$ \\
\hline GT02 & $\begin{array}{l}\text { Analizar las actividades sobre tablas y gráficos estadísticos en 17 colecciones de libros de } \\
\text { texto de matemáticas recomendadas por PNLD 2004 para los grados iniciales de la escuela } \\
\text { primaria. }\end{array}$ \\
\hline GT03 & $\begin{array}{l}\text { Analizar las propuestas relacionadas con el trabajo referente a estadística que involucran } \\
\text { gráficos y tablas en cinco colecciones de libros de texto de matemáticas de los primeros años. }\end{array}$ \\
\hline GT04 & $\begin{array}{l}\text { Analizar el tiempo sugerido y las acciones didácticas promovidas en la enseñanza de las } \\
\text { actividades estadísticas sobre tablas y gráficos en algunos libros mexicanos de nivel primaria, } \\
\text { secundaria y bachillerato. }\end{array}$ \\
\hline
\end{tabular}

Fuente: Elaboración propia con base en la revisión sistemática.

Además, a partir de los objetivos y su lectura a profundidad, es posible señalar otros fenómenos de interés considerados por los investigadores, identificando los siguientes:

- Comparativos curriculares: en este tipo de estudios se catalogan aquellos que exploran la relación entre las directrices curriculares y lo presentado en los libros de texto, de manera que en sus resultados presentan concurrencia o divergencia entre estos (por ejemplo, G04).

- Exploración de la calidad las actividades: este tipo de estudios exploran la calidad de las actividades sobre gráficos y tablas, apoyados en taxonomías, complejidad de las tareas presentes u otros resultados de la investigación, y generalmente valoran la riqueza o pobreza matemática que presentan (por ejemplo, G06).

- Tipo de representación y su complejidad: este tipo de estudios solo cuantifica el número de representaciones estadísticas, así como la complejidad semiótica presente en las tareas de los libros de texto (por ejemplo, G07, T07).

- Conversión y/o tránsito entre distintos registros de representación y relaciones intra-matemáticas: en esta categoría se han considerado aquellos estudios que exploran la traducción o conversión entre distintos registros de representación, y aquellos que hablan de la matemática que debiera movilizar el estudiantado para acceder a desarrollar alguno de los procesos propuestos en las tareas (por ejemplo, T09).

Como se puede observar, la mayoría de las investigaciones se sitúan en identificar el tipo de representación estadística y las complejidades asociadas a ellas, seguidas de aquellas que exploran el tipo de tareas que se demandan en las actividades, o bien, en la intersección de estas. A continuación, se desarrolla una reflexión aún más profunda de lo anterior, identificando las características puntuales en las que se ha centrado el análisis de las investigaciones seleccionadas. 
Con respecto a la pregunta P2: ¿Qué características se han estudiado en las investigaciones sobre tablas y gráficos estadísticos en libros de texto de educación primaria de Iberoamérica?, en la Tabla 4 se presenta el conglomerado de características, denominadas como unidades de análisis, que han considerado los investigadores en sus estudios.

TABLA 4

Unidades y categorías de análisis consideradas en las investigaciones seleccionadas.

\begin{tabular}{|c|c|}
\hline Unidad de análisis & Categorías de análisis \\
\hline Tipo de tabla (TT) & Tabla de datos (banco de datos), tabla de conteo, tabla de frecuencia, tabla de doble entrada, cuadro. \\
\hline Tipo de gráfico (TG) & $\begin{array}{l}\text { Gráfico de barras, gráfico de líneas, pictograma, gráficos de sectores, diagrama de puntos, líneas } \\
\text { múltiples, barras múltiples, mapa temático, tallo y hoja, diagrama de dispersión, histograma, gráfico } \\
\text { rectangular, climograma, polígono de frecuencia, barras bidireccionales. }\end{array}$ \\
\hline Tipo de datos (TD) & Real, ficticio. \\
\hline Nivel de lectura (NL) & $\begin{array}{l}\text { N1. Leer los datos. N2. Leer dentro de los datos. N3. Leer más allá de los datos. N4. Leer detrás de los } \\
\text { datos. }\end{array}$ \\
\hline $\begin{array}{l}\text { Nivel de complejidad } \\
\text { semiótica (NS) }\end{array}$ & $\begin{array}{l}\text { N1. Representación de datos individuales. N2. Representación de un conjunto de datos sin formar } \\
\text { su distribución. N3. Representación de una distribución de datos. N3.1. Tablas de distribución } \\
\text { de frecuencias ordinarias (absolutas, relativas o porcentuales). N3.2. Tablas de distribución de } \\
\text { frecuencias que incluyen frecuencias acumuladas (absolutas, relativas o porcentuales). N3.3. Tablas } \\
\text { donde se considera la agrupación de los valores de la variable en intervalos. N4. Representación } \\
\text { de varias distribuciones sobre una misma representación. N4.1. Tablas de doble entrada o } \\
\text { contingencias de frecuencias ordinarias (absolutas, relativas o porcentuales). N4.2. Tablas de doble } \\
\text { entrada o contingencias donde se considera la agrupación de los valores de la variable en intervalo } \\
\text { para cualquier tipo de frecuencia. }\end{array}$ \\
\hline $\begin{array}{l}\text { Actividad solicitada } \\
\text { (AS) }\end{array}$ & $\begin{array}{l}\text { Leer, construir, calcular, ejemplo, comparar, justificar, traducir, explicar, ejemplificar, recoger } \\
\text { datos, completar, buscar información, presentar información, asignar variable, inventar problema, } \\
\text { formular preguntas, e interpretar. } \\
\text { Cabe señalar que esta unidad de análisis en las investigaciones también se presenta con el nombre de } \\
\text { tarea solicitada o habilidad explorada. }\end{array}$ \\
\hline Tipo de Variable (TV) & Cualitativa ordinal, cualitativa nominal, cuantitativa discreta, cuantitativa continua. \\
\hline $\begin{array}{l}\text { Tipo de Contexto } \\
\text { (TC) }\end{array}$ & Personal, profesional/laboral, social, científico. \\
\hline $\begin{array}{l}\text { Forma de trabajo } \\
\text { (FT) }\end{array}$ & Individual, grupal. \\
\hline $\begin{array}{l}\text { Conflicto semiótico } \\
\text { (CS) }\end{array}$ & $\begin{array}{l}\text { Ausencia de título, ausencia de títulos y rótulos en los ejes, errores de proporcionalidad, uso de la } \\
\text { tercera dimensión, errores en la escala del gráfico, otros conflictos semióticos potenciales. }\end{array}$ \\
\hline $\begin{array}{l}\text { Contenidos u } \\
\text { objetos matemáticos } \\
\text { (CO) }\end{array}$ & $\begin{array}{l}\text { Localización del valor de la frecuencia de una categoría, localización de puntos máximos, } \\
\text { localización de puntos mínimos, suma total de valores, localización de una categoría a partir del } \\
\text { valor de la frecuencia, variable y valores, relación de equivalencia, funciones, funcional lineal } \\
\text { y proporcionalidad, intervalos de valores y extremos, función de dos variables, desigualdades, } \\
\text { incógnitas, expresiones simbólicas. }\end{array}$ \\
\hline $\begin{array}{l}\text { Análisis } \\
\text { ontosemiótico (AO) }\end{array}$ & $\begin{array}{l}\text { Análisis ontosemiótico sobre los objetos y significados matemáticos que se movilizan en el } \\
\text { enunciado de las tareas. }\end{array}$ \\
\hline $\begin{array}{l}\text { Propósito del gráfico } \\
\text { dentro de la tarea } \\
\text { (PG) }\end{array}$ & Propósito de análisis, de comunicación y de construcción. \\
\hline
\end{tabular}

Fuente: Elaboración propia con base en la revisión sistemática.

Como se observa, los investigadores se han enfocado en distintas unidades de análisis, por lo que se destacan aquellas relacionadas con la estructura de la representación (tipo de tabla, tipo de gráfico, tipo de variable, nivel de complejidad semiótica, conflicto semiótico), con la información representada (tipo de datos, tipo de contexto), con la demanda de las tareas (nivel de lectura, actividad solicitada, análisis ontosemiótico, contenidos u objetos matemáticos) y con la resolución de la actividad (forma de trabajo). 
En relación con la pregunta P3: ¿Cuáles son los resultados relevantes encontrados en las investigaciones rastreadas?, la Tabla 5 muestra las distintas unidades de análisis que los investigadores utilizaron como lente para analizar las actividades en las que intervienen tablas y gráficos, de las cuales se destacan: actividad solicitada (AS, 29 de 39), nivel de lectura (NL, 19 de 39), nivel de complejidad semiótica (NS, 15 de 39) y tipo de contexto (TC, 8 de 39).

TABLA 5

Unidades de análisis consideradas en las investigaciones seleccionadas.

\begin{tabular}{|c|c|c|c|c|c|c|c|c|c|c|c|c|c|}
\hline Código & TT & TG & TD & NL & NS & AS & TV & TC & FT & CS & $\mathrm{CO}$ & AO & PG \\
\hline G01 & & $x$ & & & & & & & & & $x$ & & \\
\hline G02 & & $x$ & & & & $x$ & & & & & & & \\
\hline G03 & & $x$ & & $x$ & & & & & & & & & \\
\hline G04 & & $x$ & & & & & $x$ & $x$ & & & & & \\
\hline G05 & & $x$ & & $x$ & $x$ & $x$ & & & & & & & \\
\hline G06 & & $x$ & & $x$ & $x$ & $x$ & & & & & & & \\
\hline G07 & & $x$ & & $x$ & $x$ & $x$ & & & & & & & \\
\hline G08 & & $x$ & & $x$ & & $x$ & & & & & & & \\
\hline G09 & & $x$ & & & & $x$ & & & & & & & \\
\hline G10 & & $x$ & & $x$ & $x$ & $x$ & & & & & & & \\
\hline G11 & & $x$ & & & & $x$ & & & & & & & \\
\hline G12 & & & & & & & & & & $x$ & & & \\
\hline G13 & & $x$ & & $x$ & $x$ & $x$ & & & & & & & \\
\hline G14 & & $x$ & & $x$ & $x$ & $x$ & & & & & & & \\
\hline G15 & & & & & & & & & & $x$ & & & \\
\hline G16 & & & & & & & & & & & & $x$ & \\
\hline G17 & & $x$ & & & & & & & & & & & \\
\hline G18 & & $x$ & & $x$ & $x$ & $x$ & & & & & & & \\
\hline G19 & & & & $x$ & $x$ & $x$ & & & & & & & \\
\hline G20 & & $x$ & & $x$ & $x$ & $x$ & & $x$ & & & & & \\
\hline G21 & & $x$ & & & & $x$ & $x$ & & & & & & \\
\hline G22 & & $x$ & & $x$ & $x$ & $x$ & & $x$ & & & & & $x$ \\
\hline G23 & & $x$ & & $x$ & $x$ & $x$ & & $x$ & & & & & \\
\hline G24 & & $x$ & & $x$ & $x$ & & & & & $x$ & & & \\
\hline T01 & $x$ & & & $x$ & & $x$ & $x$ & $x$ & & & & & \\
\hline T02 & $x$ & & $x$ & & & $x$ & & & & & & & \\
\hline T03 & $x$ & & & & & $x$ & & & & & & & \\
\hline T04 & & & & & & $x$ & $x$ & $x$ & $x$ & & & & \\
\hline T05 & $x$ & & & & & $x$ & & & & & & & \\
\hline T06 & $x$ & & & & & $x$ & & & & & & & \\
\hline T07 & $x$ & & & $x$ & $x$ & $x$ & & $x$ & & & & & \\
\hline T08 & $x$ & & & $x$ & & $x$ & & & & & & & \\
\hline T09 & & & & & & & & & & & & $x$ & \\
\hline T10 & $x$ & & & & $x$ & & & & & & & $x$ & \\
\hline T11 & $x$ & & & $x$ & $x$ & $x$ & & $x$ & & & & & \\
\hline GT01 & $x$ & $x$ & & & & $x$ & & & & & & & \\
\hline
\end{tabular}




\begin{tabular}{|c|c|c|c|c|c|c|c|c|c|c|c|c|c|}
\hline Código & TT & TG & TD & NL & NS & AS & TV & TC & FT & CS & CO & AO & PG \\
\hline GT02 & $\mathrm{x}$ & $\mathrm{x}$ & & & & $\mathrm{x}$ & & & & & & & \\
\hline GT03 & & & & & & $\mathrm{x}$ & & & & & & & \\
\hline GT04 & & $\mathrm{x}$ & & $\mathrm{x}$ & & $\mathrm{x}$ & & & & & & & \\
\hline Total & $\mathbf{1 1}$ & $\mathbf{2 3}$ & $\mathbf{1}$ & $\mathbf{1 9}$ & $\mathbf{1 5}$ & $\mathbf{2 9}$ & $\mathbf{4}$ & $\mathbf{8}$ & $\mathbf{1}$ & $\mathbf{3}$ & $\mathbf{1}$ & $\mathbf{3}$ & $\mathbf{1}$ \\
\hline
\end{tabular}

Teniendo en cuenta las unidades de análisis destacadas (tipo de tabla, tipo de gráfico, actividad solicitada, nivel de lectura, nivel de complejidad semiótica y tipo de contexto), en la Tabla 6 se muestra la categoría predominante encontrada en los resultados de las investigaciones del estudio.

TABLA 6

Resultados encontrados en las investigaciones seleccionadas considerando las unidades de análisis destacadas.

\begin{tabular}{|c|c|c|c|c|c|c|}
\hline Código & TT & TG & NL & NS & AS & TC \\
\hline G01 & & Barras & & & & \\
\hline G02 & & Barras & & & Leer & \\
\hline G03 & & Circular & Nivel 3 & & & \\
\hline G04 & & Barras & & & & Persona \\
\hline G05 & & Barras & Nivel 2 & Nivel 3 & $\begin{array}{l}\text { Comparar y } \\
\text { justificar }\end{array}$ & \\
\hline G06 & & Barras & Nivel 2 & Nivel 3 & Calcular & \\
\hline G07 & & Barras & Nivel 2 & Nivel 3 & Leer & \\
\hline G08 & & Barras & Nivel 1 & & Construir & \\
\hline G09 & & Barras & & & Comparar & \\
\hline G10 & & Barras & Nivel 2 & Nivel 3 & Calcular & \\
\hline G11 & & Barras & & & Leer & \\
\hline G13 & & Barras & Nivel 1 & Nivel 2 & Explicar & \\
\hline G14 & & Barras & Nivel 2 & Nivel 2 & Calcular & \\
\hline G17 & & Barras & & & & \\
\hline G18 & & Barras & Nivel 2 & Nivel 3 & Calcular & \\
\hline G19 & & & Nivel 2 & Nivel 3 & Interpretar & \\
\hline G20 & & Barras & Nivel 2 & Nivel 3 & Calcular & Personal \\
\hline G21 & & Barras & & & Interpretar & \\
\hline G22 & & Barras & Nivel 2 & Nivel 3 & Leer & Laboral \\
\hline G23 & & $\begin{array}{c}\text { Barras y } \\
\text { pictograma }\end{array}$ & Nivel 2 & Nivel 3 & Completar & Personal \\
\hline G24 & & Barras & Nivel 2 & Nivel 3 & & \\
\hline T01 & Conteo & & Nivel 2 & & Calcular & Personal \\
\hline T02 & Cuadro & & & & Llenar & \\
\hline T03 & Conteo & & & & Traducir & \\
\hline T04 & & & & & Interpretar & Personal \\
\hline T05 & Frecuencia & & & & Leer & \\
\hline T06 & Frecuencia & & & & Interpretar & \\
\hline T07 & Datos & & Nivel 2 & Nivel 2 & Calcular & Personal \\
\hline T08 & Una entrada & & Nivel 3 & & Interpretar & \\
\hline T10 & Frecuencia & & & Nivel 3 & & \\
\hline
\end{tabular}




\begin{tabular}{|c|c|c|c|c|c|c|}
\hline Código & TT & TG & NL & NS & AS & TC \\
\hline T11 & $\begin{array}{c}\text { Distribución de } \\
\text { una variable }\end{array}$ & & Nivel 2 & Nivel 3.1 & Calcular & Personal \\
\hline GT01 & Datos & Barras & & & Llenar & Organizar \\
\hline GT02 & Datos & Barras & & & Llenar & \\
\hline GT03 & & & & & Leer & \\
\hline GT04 & Frecuencia & Barras & Nivel 2 & & \\
\hline
\end{tabular}

De lo anterior, se señala que en la mayoría de las investigaciones sobre tablas predomina la tabla de frecuencias, la actividad calcular, el nivel 2 de lectura, el nivel 3 de complejidad semiótica y el contexto personal. En el caso de las investigaciones sobre gráficos, se destaca el gráfico de barras y las mismas categorías predominantes de las otras unidades de análisis en las tablas. Una diferencia notable es que el tipo de contexto ha sido poco explorado en las investigaciones sobre gráficos; mientras que, en el caso de las tablas, los niveles de lectura y complejidad semiótica.

\section{SÍNTESIS Y REFLEXIONES FINALES}

El análisis de las investigaciones seleccionadas a partir de la revisión sistemática de literatura aporta un panorama general respecto a las investigaciones que estudiaron las tablas y gráficos estadísticos en libros de texto de educación primaria de Iberoamérica. Se identificaron 39 estudios primarios que permiten apreciar el interés que han tenido los investigadores sobre esta temática desde 2006 hasta la actualidad (24 toman como objeto de estudio los gráficos, 11 las tablas y 4 ambas representaciones estadísticas); publicados en revistas y memorias de congreso (29 y 10, respectivamente). Los países iberoamericanos que se han considerado para el análisis de los libros de texto son: Chile (14 estudios), Brasil (7), España (4), Perú (3), México (3), Venezuela (2), Argentina (1), Costa Rica (1), Colombia (1), Venezuela-Guatemala (1), España-Chile (1) y Brasil-Chile (1). Con esto se evidencia que existen países de lberoamérica en donde no se ha explorado esta línea de investigación, lo cual puede llevarnos de manera natural a cuestionarnos: ¿cuáles son los motivos por los que estos países no han sido del interés de los investigadores en educación estadística?, ¿qué impacto han tenido este tipo de investigaciones en aquellos países donde se ha concentrado la investigación? Lo que no cabe duda es que, los libros de texto han servido como un informante del estado de desarrollo que guardan estos países respecto a las tablas y gráficos, representaciones consideradas como parte de la cultura estadística.

Por otro lado, la revisión permitió identificar que en la mayoría de las investigaciones se declara el objetivo analizar (26 de 39 estudios). Además, después de desarrollar una lectura a profundidad de estos, se identificaron otros cuatro focos de interés, a saber: 1) comparativos curriculares, 2) exploración de la calidad las actividades, 3) tipo de representación y su complejidad, y 4) conversión y/o tránsito entre distintos registros de representación y relaciones intra-matemáticas; siendo el segundo y tercer foco aquellos que han ocupado el mayor interés de los investigadores. Por ello, se considera interesante, como implicación a futuro, tratar de desarrollar estudios que conjunten los cuatro focos de interés, ya que de manera más clara podrían entregar resultados o herramientas a los organismos encargados de la administración, regulación y fomento de la educación de cada país, y al profesorado para el diseño e implementación de actividades sobre lectura y construcción de tablas y gráficos que fomenten el desarrollado de la cultura estadística en el estudiantado.

En una exploración con mayor profundidad, se identificaron las diversas unidades de análisis que permiten reconocer el tratamiento de las tablas y gráficos como objeto de estudio en los libros de 
texto de educación primaria, siendo las de mayor frecuencia: tipo de tabla, tipo de gráfico, actividad solicitada, nivel de lectura, nivel de complejidad semiótica y tipo de contexto. En relación con los resultados relevantes encontrados en las investigaciones rastreadas, se observa el predominio de la tabla de frecuencias, el gráfico de barras, la actividad calcular (implica encontrar un valor o dar respuesta a una pregunta, a partir de la realización de cálculos matemáticos), el nivel 2 de lectura (leer dentro de los datos, implica leer los datos y realizar comparaciones u operaciones matemáticas con ellos para determinar valores solicitados), el nivel 3 de complejidad semiótica (representación de una distribución de datos, los datos están representados en forma ordenada y se usa la idea de frecuencia) y el contexto personal (la situación se enfoca en actividades propias del estudiantado como juegos, viajes, compras, entre otras). Este resultado permite identificar aquellas unidades de análisis que han sido poco estudiadas por los investigadores, y que se consideran de importancia, por ejemplo, los conflictos semióticos que presentan los gráficos estadísticos, los cuales, sino son identificados por el profesorado en la clase de matemáticas, conllevan a concebir falsas impresiones u opiniones equivocadas en el estudiantado.

Sobre estos resultados, se considera necesario que en los libros de texto de educación primaria de los distintos países se incluyan tareas (actividades solicitadas) que propicien en el estudiantado alcanzar el nivel 3 (leer más allá de los datos) y 4 (leer detrás de los datos) de lectura, ligados a desarrollar la capacidad de predecir y evaluar críticamente información estadística, y la capacidad de discutir y comunicar tal información, y con ello, contribuir a la formación del estudiantado estadísticamente cultos. Además, se observa la necesidad de incluir tablas y gráficos relacionados con el nivel 4 de complejidad semiótica, es decir, representaciones que exhiban al menos dos distribuciones de frecuencias. Asimismo, se sugiere incluir actividades situadas en los otros tipos de contextos (social, laboral y científico) para potencializar la diversidad del tipo de información que se presenta de manera sintetizada en tablas y gráficos estadísticos.

\section{Agradecimiento}

Este trabajo ha sido desarrollado en el marco del Proyecto Fondecyt 1200005 financiado por la Agencia Nacional de Investigación y Desarrollo (ANID) de Chile.

\section{REFERENCIAS}

Arteaga, P., Batanero, C., Cañadas, G., y Contreras, J. (2011). Las tablas y gráficos estadísticos como objetos culturales. Números. Revista de Didáctica de las Matemáticas, 76, 55-67.

Arteaga, P., Díaz-Levicoy, D., y Batanero, C. (2018). Investigaciones sobre gráficos estadísticos en Educación Primera: revisión de la literatura. Revista digital: Matemática, Educación e Internet, 18(1). https://doi.org/10.18845/rdmei.v18i1.3255

Batanero, C. (2004). Los retos de la cultura estadística. Yupana. Revista de Educación Matemática de la UNL, 1, 27-36. https://doi.org/10.14409/yu.v1i1.238

Carrizo, D., y Ortiz, C. (2016). Modelos del proceso de educación de requisitos: Un mapeo sistemático. Ingeniería y desarrollo, 34(1), 184-203.

Claros, J., Sánchez, M., y Coriat, M. (2016). Tratamiento del límite finito en libros de texto españoles de secundaria: 1933-2005. Educación matemática, 28(1), 125-152.

Contreras, J. M., y Molina-Portillo, E. (2019). Alfabetización estadística. 25 años de la evolución de un término. Números: Revista de didáctica de las matemáticas, 100, 35-38. 
Curcio, F. R. (1989). Developing graph comprehension. Reston: National Council of Teachers of Mathematics [NCTM].

Del Pino, G., y Estrella, S. (2012). Educación estadística: relaciones con la matemática. Pensamiento Educativo, 49(1), 53-64.

Estrella, S. (2014). El formato tabular: una revisión de literatura. Actualidades Investigativas en Educación, 14(2), 449-478.

Fernández, M., y Caballero, P. (2017). El libro de texto como objeto de estudio y recurso didáctico para el aprendizaje: fortalezas y debilidades. Revista Electrónica Interuniversitaria de Formación del Profesorado, 20(1), 201-217.

Ferreira, A., y Mayorga, L (2010). Propuesta para la evaluación de libros de matemática de todos los niveles educativos. Revista Ciencias de la Educación, 20(35), 15-28.

Friel, S., Curcio, F. R., y Bright, G. (2001). Making sense of graphs: critical factors influencing comprehension and instructional implications. Journal for Research in Mathematics Education, 32(2), 124-158.

Gal, I. (2002). Adult's statistical literacy: Meaning, components, responsibilities. International Statistical Review, 70(1), 1-25. https://doi.org/10.1111/j.1751-5823.2002.tb00336.x

García-García, J. I., Díaz-Levicoy, D., Vidal-Henry, S., y Arredondo, E. H. (2019). Las tablas estadísticas en libros de texto de Educación Primaria en México. Revista Paradigma, 40(2), 153-175.

García-García, J. I., Encarnación, E., y Arredondo, E. H. (2020). Exploración de la comprensión gráfica de estudiantes de secundaria. IE Revista de Investigación Educativa de la REDIECH, 11, e925. https:// doi.org/10.33010/ie_rie_rediech.v11i0.925

Guirao, J. (2015). Utilidad y tipos de revisión de literatura. ENE, Revista de Enfermería, 9(2). https:// dx.doi.org/10.4321/S1988-348X2015000200002

Moreno-Gómez, F. (2021). La importancia de la revisión de la literatura en la investigación formativa. Salutem Scientia Spiritus, 7(1), 1-13.

Revelo, O., Collazos, C., y Jiménez, J. (2018). El trabajo colaborativo como estrategia didáctica para la enseñanza/aprendizaje de la programación: una revisión sistemática de literatura. Revista TecnoLógicas, 21(41), 115-134. https://doi.org/10.22430/22565337.731

Rodríguez, J. (2007). La investigación sobre los libros de texto y materiales curriculares. En Ministerio de Educación de Chile (Ed.), Primer Seminario Internacional de Textos Escolares. SITE 2006 (pp. 185-191). Santiago: MINEDUC.

Shaughnessy, J. M. (2007). Research on statistical learning and reasoning. En F. K. Lester (Ed.), Second handbook of research on mathematics teaching and learning (pp. 957-1009). Reston: National Council of Teachers of Mathematics [NCTM].

Soaje de Elias, R. (2018). Textos escolares: consideraciones didácticas. Educación y Educadores, 21(1), 73-92. https://doi.org/10.5294/edu.2018.21.1.4

Son JW., y Diletti J. (2017). What Can We Learn from Textbook Analysis? En Son JW., Watanabe T., y Lo JJ. (Eds.) What Matters? Research Trends in International Comparative Studies in Mathematics Education (pp. 3-32). Cham: Springer. https://doi.org/10.1007/978-3-319-51187-0_1

Vásquez, C., y Alsina, Á. (2014). Enseñanza de la Probabilidad en educación primaria. Un desafío para la formación inicial y continua del profesorado. Números. Revista de Didáctica de las Matemáticas, $85,5-23$. 


\section{Anexo: Referencias de las investigaciones obtenidas por la revisión sistemática}

\begin{tabular}{|c|c|}
\hline Código & Investigación \\
\hline G01 & $\begin{array}{l}\text { Lemos, M. P. F. (2006). O estudo do tratamento da informação nos livros didáticos das séries } \\
\text { iniciais do ensino fundamental. Ciência \& Educação, 12(2), 171-184. https://doi.org/10.1590/ } \\
\text { S1516-73132006000200005 }\end{array}$ \\
\hline G02 & $\begin{array}{l}\text { Arteaga, P, Ortiz, J., y Batanero, C. (2013). Un estudio de la presentación de los gráficos estadísticos } \\
\text { en libros de texto españoles de educación primaria. En R. Flores (Ed.), Acta Latinoamericana de } \\
\text { Matemática Educativa } 18 \text { (pp. 41-50). CLAME. }\end{array}$ \\
\hline G03 & $\begin{array}{l}\text { Jesus, D. S., Fernandes, J. A., y Leite. L. (2013). Relevância dos gráficos estatísticos nos manuais } \\
\text { escolares da disciplina de ciências físico-químicas. En J. A. Fernandes, F. Viseu, M. H. Martinho, y P. } \\
\text { F. Correia (Eds.), Atas do III Encontro de probabilidades e estatística na escola (pp. 145-162). Braga: } \\
\text { Centro de Investigação em Educação da Universidade do Minho. }\end{array}$ \\
\hline G04 & $\begin{array}{l}\text { Mateus, L. (2014). Estudio de gráficos estadísticos usados en una muestra de libros de matemáticas } \\
\text { para la educación básica y media en Bogotá. En L. Andrade (Ed.), Memorias del I Encuentro } \\
\text { Colombiano de Educación Estocástica (pp. 274-280). Bogotá: Asociación Colombiana de } \\
\text { Educación Estocástica. }\end{array}$ \\
\hline G05 & $\begin{array}{l}\text { Díaz-Levicoy, D., y Arteaga, P. (2014). Análisis de gráficos estadísticos en textos escolares de } \\
\text { séptimo básico en Chile. Revista electrónica: Diálogos educativos, 14(28), 21-40. }\end{array}$ \\
\hline G06 & $\begin{array}{l}\text { Díaz-Levicoy, D., Batanero, C., Arteaga, P., y López-Martín, M. (2015). Análisis de los gráficos } \\
\text { estadísticos presentados en libros de texto de educación primaria chilena. Educaçao Matematica } \\
\text { Pesquisa, 17(4), 715-739. }\end{array}$ \\
\hline G07 & $\begin{array}{l}\text { Díaz-Levicoy, D., Batanero, C., Arteaga, P., y Gea, M. M. (2015). Análisis de gráficos estadísticos en } \\
\text { libros de texto de educación primaria española. UNION. Revista Iberoamericana de Educación } \\
\text { Matemática, 40, 90-112. }\end{array}$ \\
\hline G08 & $\begin{array}{l}\text { Salcedo, A., y Ramírez, T. (2016). Gráficos estadísticos en libros de texto de matemáticas } \\
\text { venezolanos. Revista VIDYA, 36(2), 219-236. }\end{array}$ \\
\hline G09 & $\begin{array}{l}\text { Salcedo, A. (2016). Gráficos estadísticos en los libros de texto para educación primaria de } \\
\text { Guatemala y Venezuela. Educaçao Matematica Pesquisa, 18(3), 1141-1163. }\end{array}$ \\
\hline G10 & $\begin{array}{l}\text { Díaz-Levicoy, D., Batanero, C., Arteaga, P., y Gea, M. M. (2016). Gráficos estadísticos en libros de } \\
\text { texto de Educación Primaria: un estudio comparativo entre España y Chile. Bolema. Boletim de } \\
\text { Educação Matemática, 30(55), 713-737. http://dx.doi.org/10.1590/1980-4415v30n55a20 }\end{array}$ \\
\hline G11 & $\begin{array}{l}\text { Díaz-Levicoy, D., Giacomone, B., López-Martín, M., y Piñeiro, J. (2016). Estudio sobre los gráficos } \\
\text { estadísticos en libros de texto digitales de educación primaria española. Profesorado. Revista de } \\
\text { Currículum y Formación de Profesorado, 20(1), 133-156. }\end{array}$ \\
\hline G12 & $\begin{array}{l}\text { Arteaga, P., y Díaz-Levicoy, D. (2016). Conflictos semióticos sobre gráficos estadísticos en libros de } \\
\text { texto de Educación Primaria. Educação e Fronteiras On-Line, 6(17), 81-96. }\end{array}$ \\
\hline G13 & $\begin{array}{l}\text { Díaz-Levicoy, D., Pino, C., y Cruz, A. (2016). Gráficos estadísticos en libros de texto chilenos de } \\
\text { ciencias naturales. Didasc@lia: Didáctica y Educación, 7(4),75-96. }\end{array}$ \\
\hline G14 & $\begin{array}{l}\text { Díaz-Levicoy, D., Giacomone, B. y Arteaga, P. (2017). Caracterización de los gráficos estadísticos } \\
\text { en libros de texto argentinos del segundo ciclo de Educación Primaria. Profesorado. Revista de } \\
\text { Currículum y Formación del Profesorado, 21(3), 299-326. }\end{array}$ \\
\hline G15 & $\begin{array}{l}\text { Díaz-Levicoy, D., Arteaga, P., y Contreras, J. M. (2017). Conflictos semióticos potenciales sobre } \\
\text { gráficos estadísticos en libros de texto de Ciencias Naturales de Educación Primaria chilena. } \\
\text { Enseñanza de las Ciencias, (No. Extraordinario, X Congreso Internacional sobre Investigación en } \\
\text { Didáctica de las Ciencias), 905-912. }\end{array}$ \\
\hline G16 & $\begin{array}{l}\text { Díaz Levicoy, D., Godino, J. D., y Giacomone, B. (2017). Análisis Ontosemiótico de Tareas que } \\
\text { Involucran Gráficos Estadísticos en Educación Primaria. Revista Digital: Matemática, Educación e } \\
\text { Internet, 18(1). }\end{array}$ \\
\hline
\end{tabular}




\begin{tabular}{|c|c|}
\hline Código & Investigación \\
\hline G17 & $\begin{array}{l}\text { Osorio, M., y Díaz-Levicoy, D. (2018). Tipos de gráficos estadísticos en libros de texto de matemática } \\
\text { para la educación primaria peruana. Acta Latinoamericana de Matemática Educativa, 31(1), } \\
849-856 \text {. }\end{array}$ \\
\hline G18 & $\begin{array}{l}\text { Díaz-Levicoy, D., Osorio, M., Arteaga, P. y Rodríguez-Alveal, F. (2018). Gráficos estadísticos en libros } \\
\text { de texto de matemática de primaria en Perú. Bolema. Boletim de Educação Matemática, 32(61), } \\
\text { 503-525. http://dx.doi.org/10.1590/1980-4415v32n61a10 }\end{array}$ \\
\hline G19 & $\begin{array}{l}\text { Díaz-Levicoy, D., Osorio, M., Rodríguez-Alveal, F., y Ferrada, C. (2019). Los gráficos de barras en los } \\
\text { libros de texto de Educación Primaria en Perú. Paradigma, 40(1), 259-279. }\end{array}$ \\
\hline G20 & $\begin{array}{l}\text { Bustamante-Valdés, M., y Díaz-Levicoy, D. (2020). Análisis de gráficos estadísticos en módulos de } \\
\text { matemática para la enseñanza de escuelas rurales multigrado en Chile. Revista Espacios, 41(16), } \\
\text { 24-39. }\end{array}$ \\
\hline G21 & $\begin{array}{l}\text { Bustamante-Valdés, M., y Díaz-Levicoy, D. (2020). Análisis de actividades evaluativas sobre gráficos } \\
\text { estadísticos en el libro de texto del profesor para la educación rural chilena. Revista Conrado, } \\
16(77), 436-441 \text {. }\end{array}$ \\
\hline G22 & $\begin{array}{l}\text { Jiménez-Castro, M., Arteaga, P., y Batanero, C. (2020). Los gráficos estadísticos en los libros de } \\
\text { texto de Educación Primaria en Costa Rica. Bolema. Boletim de Educação Matemática, 34(66), 132- } \\
\text { 156. http://dx.doi.org/10.1590/1980-4415v34n66a07 }\end{array}$ \\
\hline G23 & $\begin{array}{l}\text { Díaz-Levicoy, D., Souza, E., y Bustamante-Valdés, M. (2020). Un estudio comparativo sobre gráficos } \\
\text { estadísticos a partir de las actividades de los libros de texto } 2^{\circ} \text { año de Educación Primaria en Brasil } \\
\text { y Chile. Areté. Revista Digital del Doctorado en Educación de la Universidad Central de Venezuela, } \\
\text { 6(12), } 111 \text { - 132. http://dx.doi.org/10.1590/1980-4415v30n55a20 }\end{array}$ \\
\hline G24 & $\begin{array}{l}\text { Vidal-Henry, S., Díaz-Levicoy, D., Navarro-Sandoval, C., y García-García, J. I. (2020). Gráficos } \\
\text { estadísticos en libros de texto de matemáticas para la Educación Primaria mexicana. Educação e } \\
\text { Fronteiras On-Line, 10(29), 153-170. }\end{array}$ \\
\hline T01 & $\begin{array}{l}\text { Díaz-Levicoy, D., Morales, R., y López-Martín, M. (2015). Tablas estadísticas en libros de texto } \\
\text { chilenos de } 1^{\circ} \text { y } 2^{\circ} \text { año de Educación Primaria. Revista Paranaense de Educação Matemática, 4(7), } \\
\text { 10-39. }\end{array}$ \\
\hline T02 & $\begin{array}{l}\text { Dias, N., y Silva, R. (2016). Apresentação e utilização de tabelas em livros didáticos de matemática } \\
\text { do } 4^{\circ} \text { e } 5^{\circ} \text { anos do ensino fundamental. EM TEIA. Revista de Educação Matemática e Tecnológica } \\
\text { Iberoamericana, } 7(1), 1-21 \text {. }\end{array}$ \\
\hline T03 & $\begin{array}{l}\text { Díaz-Levicoy, D., Ruz, F., y Molina-Portillo, E. (2017). Tablas estadísticas en libros de texto chilenos } \\
\text { de tercer año de educación primaria. Espaço Plural, 18(36), 196-218. }\end{array}$ \\
\hline T04 & $\begin{array}{l}\text { Díaz-Levicoy, D., Vásquez, C. y Molina-Portillo, E. (2018). Estudio exploratorio sobre tablas } \\
\text { estadísticas en libros de texto de tercer año de Educación Primaria. Tangram. Revista de Educação } \\
\text { Matemática, 1(2), 18-39. }\end{array}$ \\
\hline T05 & $\begin{array}{l}\text { Pallauta, J. D. y Gea, M. (2019). Las actividades sobre tablas estadísticas en textos escolares chilenos } \\
\text { de educación básica. En J. M. Contreras, M. M. Gea, M. M. López-Martín y E. Molina-Portillo (Eds.), } \\
\text { Actas del Tercer Congreso Internacional Virtual de Educación Estadística. }\end{array}$ \\
\hline T06 & $\begin{array}{l}\text { Evangelista, B., y Guimarães, G. (2019). Análise de atividades sobre tabelas em livros didáticos } \\
\text { brasileiros dos anos iniciais do Ensino Fundamental. En J. M. Contreras, M. M. Gea, M. M. López- } \\
\text { Martín y E. Molina-Portillo (Eds.), Actas del Tercer Congreso Internacional Virtual de Educación } \\
\text { Estadística. }\end{array}$ \\
\hline T07 & $\begin{array}{l}\text { García-García, J. I., Díaz-Levicoy, D., Vidal-Henry, S., y Arredondo, E. H. (2019). Las tablas estadísticas } \\
\text { en libros de texto de educación primaria en México. Revista Paradigma, 40(2), 153-175. }\end{array}$ \\
\hline T08 & $\begin{array}{l}\text { Salcedo, A. (2020). Actividades de tablas estadísticas en textos escolares de Matemáticas. Revista } \\
\text { digital: Matemática, Educación e Internet, 19(2). https://doi.org/10.18845/rdmei.v20i2.5044 }\end{array}$ \\
\hline T09 & $\begin{array}{l}\text { Pallauta, J., Gea, M. M., y Batanero, C. (2020). Análisis de la actividad algebraica implicada en el } \\
\text { trabajo con las tablas estadísticas. Revista digital: Matemática, Educación e Internet, 20(2). https:// } \\
\text { doi.org/10.18845/rdmei.v20i2.5036 }\end{array}$ \\
\hline T10 & $\begin{array}{l}\text { Pallauta, J., Gea, M. M., Batanero, C. (2020). Un análisis semiótico del objeto tabla estadística en } \\
\text { libros de texto chilenos. Zetetike, 28, e020001. https://doi.org/10.20396/zet.v28i0.8656257 }\end{array}$ \\
\hline
\end{tabular}




\begin{tabular}{|c|l|}
\hline Código & \multicolumn{1}{c|}{ Investigación } \\
\hline T11 & $\begin{array}{l}\text { Pallauta, J., Gea, M.M., y Arteaga, P. (2021). Caracterización de las Tareas propuestas sobre Tablas } \\
\text { Estadísticas en Libros de Texto Chilenos de Educación Básica. Revista Paradigma, 42(Nro. Extra 1: } \\
\text { Educación Estadística), 32-60. }\end{array}$ \\
\hline GT01 & $\begin{array}{l}\text { Guimarães, G. L., Gitirana, V., Melo, M. C. M. y Cavalcanti, M. R. G. (2006, Julio). Livro didático: análise } \\
\text { sobre representação em gráficos e tabelas. Trabajo presentado en el I Simpósio Internacional de } \\
\text { Pesquisa em Educação Matemática. Recife, Brasil. }\end{array}$ \\
\hline GT02 & $\begin{array}{l}\text { Guimarães, G., Gitirana, V., Cavalcanti, M. y Marques, M. (2008, Julio). Análise das atividades sobre } \\
\text { representações gráficas nos livros didáticos de matemática. Trabajo presentado en el II Simpósio } \\
\text { Internacional de Pesquisa em Educação Matemática. Recife, Brasil. }\end{array}$ \\
\hline GT03 & $\begin{array}{l}\text { Bivar, D. B. y Selva, A. C. (2011, Junio). Analisando atividades envolvendo gráficos e tabelas nos } \\
\text { livros didáticos de matemática. Trabajo presentado en la XIII Conferência Interamericana de } \\
\text { Educação Matemática. Recife, Brasil. }\end{array}$ \\
\hline GT04 & $\begin{array}{l}\text { Pérez, Y., Ruiz, B. y Hugues, E. (2019). Análisis de actividades estadísticas en libros de textos de } \\
\text { nivel básico y medio superior en México. Revista Digital: Matemática, Educación e Internet, 19(2). }\end{array}$ \\
\hline
\end{tabular}

